\title{
Estimation of reliability of seismic and electromagnetic monitoring in seismic active areas by diffraction tomography
}

\author{
V. N. Troyan ${ }^{1}$ and Yu. V. Kiselev ${ }^{1}$ \\ ${ }^{1}$ Institute of Physics, St. Petersburg State University, Ul'yanovskaya 1, St. Petersburg, 198904, Russia
}

Received: 15 May 2001 - Accepted: 25 June 2001

\begin{abstract}
This paper presents the algorithms and results of the numerical simulation of the solution of a 2-D inverse problem on the restoration of seismic parameters and electrical conductivity of local inhomogeneities by the diffraction tomography method based upon the first order Born approximation. The direct problems for the Lame and Maxwell equations are solved by the finite difference method. Restoration of inhomogeneities which are not very weak is implemented with the use of a small number of receivers (source-receiver pairs).
\end{abstract}

\section{Introduction}

Diffraction tomography is an imaging technique that makes use of a large volume of input data (recorded traces) to produce the image of underground medium parameters with high spatial resolution. Diffraction tomography is different from the ray tomography (travel time tomography), for which the resolution is connected to the Fresnel zone and a large number of the source-receiver pairs is required. Diffraction tomography (Devaney, 1984; Devaney and Zhang, 1991; Ryzhikov and Troyan, 1994; Kiselev and Troyan, 1999) provides information about the medium parameters with subwavelength resolution.

In our study the main attention is given to an estimation of accuracy of the restoration of elastic parameters and the restoration of an electrical conductivity, with the use of an elastic wave and an electromagnetic wave. As a tool for our investigation, we use the numerical simulation in a 2-D space domain. The direct problem is solved by the finitedifference method that allows us to take correctly into account the diffraction phenomenon produced by the target inhomogeneities.

The applicability of the first order Born approximation in diffraction tomography is shown by numerical simulation

Correspondence to: V. N. Troyan (Troyan@hq.pu.ru) conducted on a single cylinder using analytical expressions for the exact scattering field (Slaney et al., 1984).

Diffraction tomography, based on the hyperbolic type equation, produces satisfactory images for inhomogeneities which are not very weak and which have sizes comparable with a wavelength. Low frequency electromagnetic fields in the Earth are governed by the parabolic type equation (diffusion equation) (Torres-Verdin and Bostick, 1992; Zhou et al., 1993; Alumbaugh and Morrison, 1995; Wilt et al., 1995) and demonstrate possibilities for mapping of the underground.

It is probable that the embodying of the monitoring of seismic active areas, in practice, will interfere with a limited number of receivers.

2 Basic equations and tomography algorithm for anelastic case

The numerical simulation to restore the parameters of local inhomogeneities with a smooth change in the elastic parameters $\lambda$ and $\mu$, and mass density $\rho$ is carried out for a twodimensional model of the elastic medium containing the free surface and plane-parallel welded interfaces with a rigid contact.

The source $\hat{\boldsymbol{f}} \equiv \hat{\boldsymbol{f}}(\boldsymbol{x}, t)$, located in the point $\left(x=x_{s}, z=\right.$ $\left.z_{s}\right)$ of the Cartesian system of coordinates $\left(x, y, z ; \boldsymbol{e}_{1}, \boldsymbol{e}_{2}, \boldsymbol{e}_{3}\right)$, produces the wave field $\boldsymbol{u} \equiv \boldsymbol{u}(x, z, t) \equiv \boldsymbol{u}(\boldsymbol{x}, t)$ which satisfies the equation

$$
\begin{aligned}
L \boldsymbol{u} & =-\hat{\boldsymbol{f}}, \quad L \boldsymbol{u} \equiv(\lambda+\mu) \nabla \nabla \cdot \boldsymbol{u}+\mu \Delta \boldsymbol{u}+\nabla \lambda \nabla \cdot \boldsymbol{u} \\
& +\nabla \mu \times \boldsymbol{u}+2(\nabla \mu \cdot \nabla) \boldsymbol{u}-\rho \frac{\partial^{2} \boldsymbol{u}}{\partial t^{2}} .
\end{aligned}
$$

We introduce the differences $\delta \lambda(\boldsymbol{x})=\lambda(\boldsymbol{x})-\lambda_{\mathrm{r} f}(\boldsymbol{x})$, $\delta \mu(\boldsymbol{x})=\mu(\boldsymbol{x})-\mu_{\mathrm{r} f}(\boldsymbol{x})$ and $\delta \rho(\boldsymbol{x})=\rho(\boldsymbol{x})-\rho_{\mathrm{r} f}(\boldsymbol{x})$ of the values $\lambda(\boldsymbol{x}), \mu(\boldsymbol{x}), \rho(\boldsymbol{x})$ for the unknown medium which are connected to the wave field $\boldsymbol{u}(\boldsymbol{x}, t)$, described by Eq. (1), and the values $\lambda_{\mathrm{rf}}(\boldsymbol{x}), \mu_{\mathrm{rf}}(\boldsymbol{x}), \rho_{\mathrm{rf}}(\boldsymbol{x})$ for the known reference 
(rf) medium for which the wave field $\boldsymbol{u}_{\mathrm{rf}}(\boldsymbol{x}, t)$ satisfies the equation

$L_{\mathrm{rf}} \boldsymbol{u}_{\mathrm{rf}}=-\hat{\boldsymbol{f}}$.

Assuming $\delta \lambda, \delta \mu$ and $\delta \rho$ are small, in accordance with the first order Born approximation, we can write

$L_{\mathrm{rf}} \delta \boldsymbol{u} \approx-\delta L \boldsymbol{u}_{\mathrm{rf}}$,

where $\delta \boldsymbol{u}=\boldsymbol{u}-\boldsymbol{u}_{\mathrm{rf}}$ is the difference field. The right-hand side of Eq. (3) is given by

$$
\begin{gathered}
\delta L \boldsymbol{u}_{\mathrm{rf}}=\boldsymbol{\nabla} \times\left(\delta \mu \nabla \times \boldsymbol{u}_{\mathrm{rf}}\right)+2 \sum_{j=1,3} \nabla \cdot\left(\delta \mu \nabla u_{\mathrm{rf} j}\right) \boldsymbol{e}_{j} \\
+\boldsymbol{\nabla}\left(\delta \lambda \boldsymbol{\nabla} \cdot \boldsymbol{u}_{\mathrm{rf}}\right)-\delta \rho \frac{\partial^{2} \boldsymbol{u}_{\mathrm{rf}}}{\partial t^{2}}
\end{gathered}
$$

can be considered as a source of that field.

We shall represent the components of the difference field $\delta u_{i}$ from Eq. (3) at the observation point of $\boldsymbol{x}=\boldsymbol{x}_{r}$, as follows

$$
\begin{aligned}
& \delta u_{i}\left(\boldsymbol{x}_{s}, \boldsymbol{x}_{r}, t\right)=\int_{S} \int_{0}^{\infty} \tilde{\boldsymbol{u}}_{i}\left(\boldsymbol{x}, \boldsymbol{x}_{r}, t-\tau\right) \\
& \cdot \delta L \boldsymbol{u}\left(\boldsymbol{x}, \boldsymbol{x}_{s}, \tau\right) d \tau d \boldsymbol{x},
\end{aligned}
$$

where $S$ is the region of restoration; $\boldsymbol{u} \equiv \boldsymbol{u}\left(\boldsymbol{x}, \boldsymbol{x}_{s}, t\right)$ and $\tilde{\boldsymbol{u}}_{1} \equiv \tilde{\boldsymbol{u}}_{1}\left(\boldsymbol{x}, \boldsymbol{x}_{r}, t\right), \tilde{\boldsymbol{u}}_{3} \equiv \tilde{\boldsymbol{u}}_{3}\left(\boldsymbol{x}, \boldsymbol{x}_{r}, t\right)$ are the solutions of Eq. (1) and Eq. (2) with the source functions

$\hat{\boldsymbol{f}} \equiv \hat{\boldsymbol{f}}\left(x_{s}, z_{s}, t\right)=\delta\left(x-x_{s}\right) \delta\left(z-z_{s}\right) f(t) \boldsymbol{e}_{3}$

and

$\hat{\boldsymbol{f}} \equiv \hat{\boldsymbol{f}}_{1} \equiv \hat{\boldsymbol{f}}_{1}\left(x_{r}, z_{r}, t\right)=\delta\left(x-x_{r}\right) \delta\left(z-z_{r}\right) \delta(t) \boldsymbol{e}_{1}$,

$\hat{\boldsymbol{f}} \equiv \hat{\boldsymbol{f}}_{3} \equiv \hat{\boldsymbol{f}}_{3}\left(x_{r}, z_{r}, t\right)=\delta\left(x-x_{r}\right) \delta\left(z-z_{r}\right) \delta(t) \boldsymbol{e}_{3}$,

respectively. The sources from Eq. (7) are located at the receiver point $\boldsymbol{x}=\boldsymbol{x}_{s}$ and have a time dependence of $\delta(t)$, which is the Dirac function.

After introducing the tomography functionals

$$
\begin{gathered}
p_{i}^{\rho}\left(\boldsymbol{x}, \boldsymbol{x}_{\boldsymbol{r}}, \boldsymbol{x}_{s}, t\right)=-\int_{0}^{\infty} \tilde{\boldsymbol{u}}_{i}\left(\boldsymbol{x}, \boldsymbol{x}_{\boldsymbol{r}}, t-\tau\right) \cdot \frac{\partial^{2}}{\partial \tau^{2}} \\
\boldsymbol{u}\left(\boldsymbol{x}, \boldsymbol{x}_{\boldsymbol{s}}, \tau\right) d \tau, \\
p_{i}^{\lambda}\left(\boldsymbol{x}, \boldsymbol{x}_{\boldsymbol{s}}, \boldsymbol{x}_{r}, t\right)=-\int_{0}^{\infty} \nabla \cdot \tilde{\boldsymbol{u}}_{i}\left(\boldsymbol{x}, \boldsymbol{x}_{\boldsymbol{r}}, t-\tau\right) \nabla \\
\cdot \boldsymbol{u}\left(\boldsymbol{x}, \boldsymbol{x}_{\boldsymbol{s}}, t-\tau\right) d \tau, \\
p_{i}^{\mu}\left(\boldsymbol{x}, \boldsymbol{x}_{\boldsymbol{s}}, \boldsymbol{x}_{r}, t\right)=+\int_{0}^{\infty}\left[\nabla \times \tilde{\boldsymbol{u}}_{i}\left(\boldsymbol{x}, \boldsymbol{x}_{\boldsymbol{r}}, t-\tau\right)\right. \\
\cdot \boldsymbol{\nabla} \times \boldsymbol{u}\left(\boldsymbol{x}, \boldsymbol{x}_{\boldsymbol{s}}, \tau\right)-2 \sum_{j=1,3} \nabla \tilde{u}_{i j}\left(\boldsymbol{x}, \boldsymbol{x}_{\boldsymbol{r}}, t-\tau\right) \\
\left.\cdot \nabla u_{j}\left(\boldsymbol{x}, \boldsymbol{x}_{\boldsymbol{s}}, \tau\right)\right] d \tau,
\end{gathered}
$$

the components of the difference field $\delta u_{i}$ described in Eq. (5) can be written down as

$$
\begin{gathered}
\delta u_{i}\left(\boldsymbol{x}_{s}, \boldsymbol{x}_{r}, t\right)=\int_{S}\left[\delta \lambda(\boldsymbol{x}) p_{i}^{\lambda}\left(\boldsymbol{x}, \boldsymbol{x}_{s}, \boldsymbol{x}_{r}, t\right)+\delta \mu(\boldsymbol{x})\right. \\
\left.p_{i}^{\mu}\left(\boldsymbol{x}, \boldsymbol{x}_{s}, \boldsymbol{x}_{r}, t\right)+\delta \rho(\boldsymbol{x}) p_{i}^{\rho}\left(\boldsymbol{x}, \boldsymbol{x}_{s}, \boldsymbol{x}_{r}, t\right)\right] d \boldsymbol{x} .
\end{gathered}
$$

Using the linear relations between $\delta \lambda(\boldsymbol{x}), \delta \rho(\boldsymbol{x})$ and $\delta \mu(\boldsymbol{x})$

$$
\begin{gathered}
\delta \lambda(\boldsymbol{x})=c_{\lambda} \delta \mu(\boldsymbol{x}), \quad \delta \rho(\boldsymbol{x})=c_{\rho} \delta \mu(\boldsymbol{x}), \\
\left(c_{\lambda}=\text { const }, \quad c_{\rho}=\text { const }\right),
\end{gathered}
$$

Eq. (9) can be rewritten as

$$
\begin{aligned}
& \delta u_{i}\left(\boldsymbol{x}_{s}, \boldsymbol{x}_{r}, t\right) \approx \int_{S}\left[c_{\lambda} p_{i}^{\lambda}\left(\boldsymbol{x}, \boldsymbol{x}_{s}, \boldsymbol{x}_{r}, t\right)+p_{i}^{\mu}\left(\boldsymbol{x}, \boldsymbol{x}_{s}, \boldsymbol{x}_{r}, t\right)\right. \\
& \left.\quad+c_{\rho} p_{i}^{\rho}\left(\boldsymbol{x}, \boldsymbol{x}_{s}, \boldsymbol{x}_{r}, t\right)\right] \delta \mu(\boldsymbol{x}) d \boldsymbol{x} .
\end{aligned}
$$

After the digitization of Eq. (11), the system of equations for the determination of $\delta \mu$ (vector $\boldsymbol{d}_{\mu}$ ), $c_{\lambda}$ and $c_{\rho}$ can be written as

$P\left(c_{\lambda}, c_{\rho}\right) \boldsymbol{d}_{\mu}=\boldsymbol{d}_{u}$,

where $\boldsymbol{d}_{u}$ are the samples of the scattered field. The final version of this system after introducing regularizing terms reads as

$$
\begin{aligned}
& {\left[P^{\prime}\left(c_{\lambda}, c_{\rho}\right) P\left(c_{\lambda}, c_{\rho}\right)+\alpha_{1}\left(B_{x}^{\prime} B_{x}+B_{z}^{\prime} B_{z}\right)+\alpha_{2} C^{\prime} C\right.} \\
& \left.\quad+\alpha_{3} D^{\prime} D\right] \boldsymbol{d}_{\mu}=P^{\prime}\left(c_{\lambda}, c_{\rho}\right) \boldsymbol{d}_{u},
\end{aligned}
$$

where $\alpha_{1}, \alpha_{2}, \alpha_{3}$ are the regularizing coefficients; matrices $B_{x}$ and $B_{z}$ are the finite difference images of second partial derivatives with respect to $x$ and $z ; C$ and $D$ are penalty matrices for non-zero values of $\boldsymbol{d}_{\mu}$ at the boundary and near the boundary points of the restored region $S$.

We find $\boldsymbol{d}_{\mu}, c_{\lambda}$ and $c_{\rho}$ by using an iterative procedure. At the first step Eq. (13) is solved with some initial values $c_{\lambda}^{(0)}$ and $c_{\rho}^{(0)}$. By minimizing the square difference of the lefthand side and the right-hand side of Eq. (12), we find $c_{\lambda}^{(1)}$ and $c_{\rho}^{(1)}$, which are the corrected values of $c_{\lambda}^{(0)}$ and $c_{\rho}^{(0)}$. At the second step, the values $c_{\lambda}^{(1)}$ and $c_{\rho}^{(1)}$ are used for the solution of the Eq. (13) system. The convergence of this procedure is based on the distinctions of the scattering diagrams ( $\mathrm{Wu}$ and Aki, 1985) created by the elementary disturbances of $\lambda, \mu$, $\rho$.

\section{Basic equations and tomography algorithm for elec- tromagnetic case}

Numerical simulation to restore the local inhomogeneities of electrical conductivity $\sigma=\sigma(\boldsymbol{x})$, located in the uniform space (electrical conductivity $\sigma=$ const, electrical permittivity $\varepsilon^{\prime}=\varepsilon \varepsilon_{0}=$ const , magnetic permittivity $\mu^{\prime}=\mu \mu_{0}=$ const) is implemented for the 2-D problem. The link between the electrical field $\boldsymbol{E}=\boldsymbol{E}(\boldsymbol{x}, t)$ and the magnetic field $\boldsymbol{H}=\boldsymbol{H}(\boldsymbol{x}, t)$ in conductive media, excited by a current density $\boldsymbol{j}_{e x}=\boldsymbol{j}_{e x}(\boldsymbol{x}, t)$, is determined by the Maxwell equations, written as

$$
\nabla \times \boldsymbol{E}=-\mu^{\prime} \frac{\partial \boldsymbol{H}}{\partial t}, \quad \nabla \times \boldsymbol{H}=\boldsymbol{j}+\boldsymbol{j}_{e x}, \quad \boldsymbol{j}=\sigma \boldsymbol{E} .
$$


Electrical field $\boldsymbol{E}=\boldsymbol{E}(\boldsymbol{x}, t)$ in the medium, containing the local inhomogeneity of $\sigma=\sigma(\boldsymbol{x})$, is given by a solution of the following equation

$$
L \boldsymbol{E}=-\frac{\partial}{\partial t} \boldsymbol{j}_{e x}, \quad L \boldsymbol{E} \equiv \frac{1}{\mu^{\prime}} \nabla \times \nabla \times \boldsymbol{E}+\sigma \frac{\partial \boldsymbol{E}}{\partial t} .
$$

The reference medium (rf) is supposed to be known $\left(\sigma_{\mathrm{rf}}\right)$ and the electrical field $\boldsymbol{E}_{\mathrm{rf}}$ satisfies the equation

$$
\begin{aligned}
L_{\mathrm{rf}} \boldsymbol{E}_{\mathrm{rf}}=-\frac{\partial}{\partial t} \boldsymbol{j}_{e x}, & \\
L_{\mathrm{rf}} \boldsymbol{E}_{\mathrm{rf}} & \equiv \frac{1}{\mu^{\prime}} \nabla \times \nabla \times \boldsymbol{E}_{\mathrm{rf}}+\sigma_{\mathrm{rf}} \frac{\partial \boldsymbol{E}_{\mathrm{rf}}}{\partial t}
\end{aligned}
$$

As in the case of scattering by elastic inhomogeneities discussed earlier, we assume that the magnitude of the value $\delta \sigma=\sigma-\sigma_{\text {rf }}$ makes it possible to write an approximate equality

$L_{\mathrm{rf}} \delta \boldsymbol{E} \approx-\delta L \boldsymbol{E}_{\mathrm{rf}}$,

where $\delta \boldsymbol{E}=\boldsymbol{E}-\boldsymbol{E}_{\mathrm{rf}}$ is the difference field. Thus, the value

$\delta L \boldsymbol{E}_{\mathrm{rf}}=\delta \sigma \frac{\partial \boldsymbol{E}_{\mathrm{rf}}}{\partial t}$

can be considered as a source of this field. With the components of the difference field $\delta E_{i}$ it is possible to write down the equation as

$$
\begin{gathered}
\delta E_{i}\left(\boldsymbol{x}_{s}, \boldsymbol{x}_{r}, t\right)=\int_{S} \int_{0}^{\infty} \widetilde{\boldsymbol{E}}_{i}\left(\boldsymbol{x}, \boldsymbol{x}_{r}, t-\tau\right) \\
. \delta L \boldsymbol{E}\left(\boldsymbol{x}, \boldsymbol{x}_{s}, \tau\right) d \tau d \boldsymbol{x} .
\end{gathered}
$$

Wave fields $\boldsymbol{E}\left(\boldsymbol{x}, \boldsymbol{x}_{s}, t\right)$ and $\widetilde{\boldsymbol{E}}_{i}\left(\boldsymbol{x}, \boldsymbol{x}_{r}, t\right)$ satisfy, respectively, the following equations

$L_{\mathrm{rf}} \boldsymbol{E}=-\hat{\boldsymbol{f}}=\delta\left(z-z_{s}\right) f(t) \boldsymbol{e}_{1}$

and

$L_{\mathrm{rf}} \widetilde{\boldsymbol{E}}_{i}=-\hat{\boldsymbol{f}}_{i} \quad(i=1,3)$,

where the sources $\hat{\boldsymbol{f}}_{i}$ are the same as in Eq. (7).

Introducing the tomography functional

$$
\begin{gathered}
p_{i}^{\sigma}\left(\boldsymbol{x}, \boldsymbol{x}_{r}, \boldsymbol{x}_{s}, t\right)=\int_{0}^{\infty} \tilde{\boldsymbol{E}}_{i}\left(\boldsymbol{x}, \boldsymbol{x}_{r}, t-\tau\right) \\
\cdot \frac{\partial}{\partial \tau} \boldsymbol{E}\left(\boldsymbol{x}, \boldsymbol{x}_{s}, \tau\right) d \tau,
\end{gathered}
$$

the components of the difference field can be written as

$\delta E_{i}\left(\boldsymbol{x}_{s}, \boldsymbol{x}_{r}, t\right) \equiv \delta E_{i}=\int_{S} \delta \sigma p_{i}^{\sigma} d \boldsymbol{x}$.

After the digitization of Eq. (23) one can write the system of linear equations

$P \boldsymbol{d}_{\sigma}=\boldsymbol{d}_{E}$

with respect to the vector $\boldsymbol{d}_{\sigma}$ which is sought for the value $\delta \sigma(\boldsymbol{x})$, where $\boldsymbol{d}_{E}$ are the time samples of the components of the wave field scattered by inhomogeneity. The final version of these equations, after introducing the regularization terms, is coincident with the system of linear equations similar to Eq. (13).

\section{Numerical simulation}

Numerical simulation on the restoration of the parameters of local inhomogeneities was implemented for a small number of receivers (3) and a small number of source receiver pairs (3-9). In the elastic case, an inhomogeneity is placed inside a piecewise homogeneous medium with plane parallel interfaces. The seismic sources which excited the wave field are located at the free surface and inside the half space. The inhomogeneities of simple and complex shapes, comparable in size with the longitudinal wavelength, are located at the depth of a 2-7 wavelength. The results of the numerical simulation are represented in Kiselev and Troyan (1998) and Kiselev and Troyan (1999). The main consequences from the numerical simulation are the following. For the inhomogeneities with contrast of $20-50 \%$ relative to the reference medium, the error of restoration is comparable with the value of contrast and the resolution is approximately half of the wavelength. The multiparameter restoration using Eq. (10) requires a high quality of input data and suitable observation schemes based on the scattering properties (Wu and Aki, 1985) of the perturbation of the medium. In the opposite case, the input data can be uninformative concerning the desired parameters. If the values $c_{\lambda}$ and $c_{\rho}$ from Eq. (10) are known, then restoration is more stable. Particularly in the case of the approximation of the desired inhomogeneity by a simple parametric function.

For the restoration of electrical conductivity as the source of the electromagnetic field, a thread with an electrical current is used. The results of restoration are represented in Fig. 1, which is obtained for an inhomogeneity (from Fig. 1a) of the size of $8 \mathrm{~km}$, with the center located at a depth of $10 \mathrm{~km}$. The maximum value of the electrical conductivity of inhomogeneity and the reference medium is equal to $10^{-4}$ $\mathrm{S} / \mathrm{m}$. An apparent frequency of the source is $10 \mathrm{~Hz}$. Restoration is implemented with the use of three receiver points: two receivers are located at a distance of $10 \mathrm{~km}$, reflecting in an origin of coordinates, and one receiver is located in an origin of coordinates. For the restoration of the electrical conductivity, we use the horizontal component of the electrical field. We do not discuss the problem of the calculation of the wave field for the reference medium (Mauriello and Patella, 1999) for the case of a field experiment. The results of the restoration for various degrees of account of a priori data are represented in Fig. 1. The best restoration (Fig. 1d) is obtained for the case where the penalty function for nonzero values of the electrical conductivity at the boundary point and near the boundary points of the region of restoration is introduced. In this case, the error of restoration is about $20 \%$. The restorations without a priori data (error $\sim 50 \%$ ) and with the penalty function for boundary points (error $\sim 40 \%$ ) are represented in Fig. 1b and Fig. 1c, respectively. Another observation scheme with smaller distance between the origin point and the two receivers leads to an increase in the error of restoration.

The estimation of accuracy of the restoration of the parameters are given by the results of the numerical simula- 

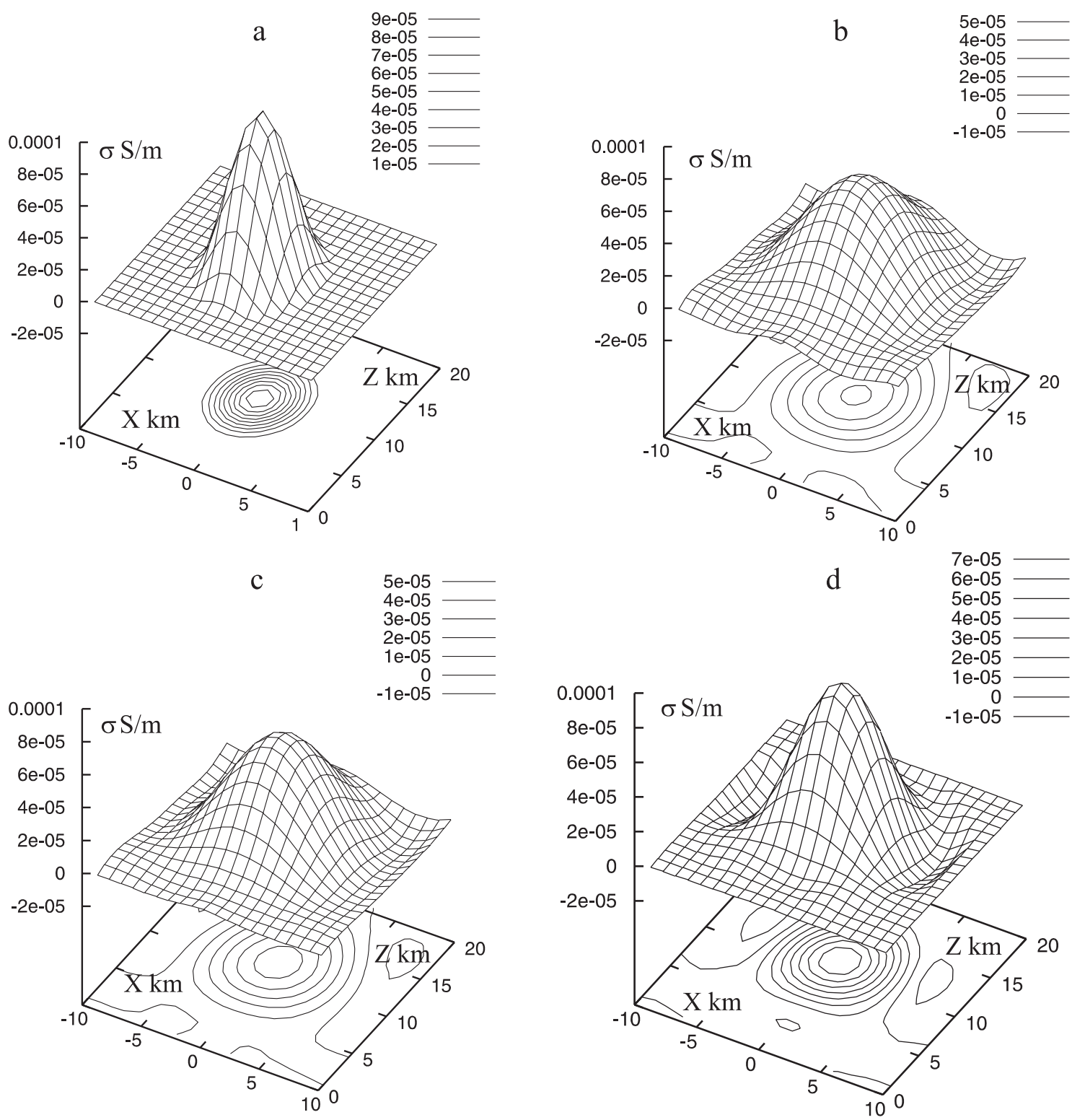

Fig. 1. Restoration of the electrical conductivity. The model (a) and the results of restoration without a priori information (b); introducing the penalty function for the boundary points (c); introducing the penalty function for nonzero values at the boundary points and near the boundary points $(\mathbf{d})$.

tion, minus a noise. The presence of the noise, which distorts the signal scattered by inhomogeneity, gives a magnification of the errors of restoration of the parameters. For example, in the case of adding an uncorrelated Gaussian noise with a standard deviation of $20 \%$ from the maximum value of the scattered signal, the magnification of the error of restoration of the parameters at 1.5-2.0 times is observed.

As in the case of the presence of the noise and in the case of the absence of the noise, this problem is ill-defined and an introduction of a regularization is required. Regarding the regularization, we consider a restriction on the magnitudes of the second partial derivatives of the parameters which are the spatial functions. The value of such a restriction is determined by the parameter of regularization $\alpha_{1}$ from Eq. (13).
We choose the magnitude of $\alpha_{1}$ so that the system of Eq. (13) has the satisfactory condition.

\section{Conclusions}

The main idea of our numerical experiments consists in the estimation of the reliability of the method of diffraction tomography for the estimation of the accuracy of the restoration of the parameters of an underground medium with the use of a small number of receivers. The diffraction tomography method (at least potentially) allows us to restore the underground seismic parameters and electrical conductivity under this condition, and this is a premise for using diffrac- 
tion tomography as a tool for monitoring seismic active areas. It is natural that a small number of input data requires the introduction of a priori information given for an increasing in the parameters for estimation accuracy.

Acknowledgements. This investigation was supported by INTAS99-1102, Russian Foundation of Basic Research (Grant 99-0564127), Ministry of Education (Grant E00-9.0-82) and Research Program 'Intergeofizika".

\section{References}

Alumbaugh, D. L. and Morrison, H. F.: Theoretical and practical consideration for crosswell electromagnetic tomography assuming a cylindrical geometry, Geophysics, 60, 846-870, 1995.

Devaney, A. J.: Geophysical diffraction tomography, IEEE Trans. Geosci. Remote Sensing, GE-22, 3-13, 1984.

Devaney, A. J. and Zhang, D.: Geophysical diffraction tomography in a layered background, Wave motion, 14, 3, 243-265, 1991.

Kiselev, Yu. V. and Troyan, V. N.: Numerical modeling in diffraction tomography, Problems of geophysics, 35, St.Petersburg Univ. Press, Russia, 25-38, 1998.
Kiselev, Yu. V. and Troyan, V. N.: Restoration of elastic and velocity parameters in diffraction tomography, Proc., of the International Seminar "Day on Diffraction-99", St.Petersburg Univ. Press, Russia, 97-102, 1999.

Mauriello, P. and Patella, D.: Principles of probability tomography for natural-source electromagnetic induction fields, Geophysics, 64, 1403-1417, 1999.

Ryzhikov, G. A. and Troyan, V. N.: Tomography and inverse sounding problems, St. Petersburg Univ. Press, Russia, 1994.

Slaney, M., Kak, A. C. and Larsen, L. E.: Limitation of imaging with first-order diffraction tomography, IEEE Trans. Microwave Theory and Tech., MTT-32, 8, 860-874, 1984.

Torres-Verdin, C. and Bostick F. X.: Implications of the Born approximation for the magnetotelluric problem in threedimensional environment, Geophysics, 57, 587-602, 1992.

Wilt, M. J., Alumbaugh, D. L., Morrison H. F., Becker, A., Lee K. H., and Deszcz-Pan M.: Crosswell electromagnetic tomography: System design considerations and field results, Geophysics, 60, 871-885, 1995.

Wu, R. and Aki, K.: Scattering characteristics of elastic waves by an elastic heterogeneity, Geophysics, 50, 585-592, 1985.

Zhou, Q., Becker A., and Morrison, H. F.: Audio-frequency electromagnetic tomography, Geophysics, 58, 482-495, 1993. 